\title{
ANÁLISIS CARACTERÍSTICO DE LOS FACTORES DE LA DESERCIÓN EN EDUCACIÓN SUPERIOR.
}

\author{
CHARACTERISTIC ANALYSIS OF THE DROPOUT FACTORS IN HIGHER \\ EDUCATION.
}

Recibido: 21 de octubre de 2021

Aceptado: 01 de diciembre de 2021
R. G. García Ramírez ${ }^{1}$

J. S. García Montejo ${ }^{2}$

\section{RESUMEN}

Este artículo presenta un análisis documental sobre la deserción; así como, las causas que la generan en las instituciones de nivel superior. Las bases de datos utilizadas fueron Science Direct, Scielo, Redalyc, Google Académico, Latindex, obteniendo una muestra de 53 documentos científicos identificados con las palabras clave "deserción escolar". Entre otros factores, se consideraron cuatro variables clave dentro del análisis, tales como: conceptualización de la deserción escolar, descripción de posibles factores de deserción escolar, caracterización de los factores de mayor impacto en la deserción escolar, programas y metodologías para abordar y disminuir la deserción escolar; mediante el proceso de revisión documental se delimitó la información para extraer únicamente la más destacada contenida en diferentes fuentes. Los resultados permitieron determinar los factores que se encuentran inmersos dentro de la deserción escolar y como es que estos forman parte de un riesgo materializado en el momento que un estudiante decide truncar su educación; es por ello que, como conclusión del presente análisis se propone que las instituciones creen mecanismos de detección e implementen modificaciones a sus programas de estudio incluyendo estrategias didácticas y seguimientos tutoriales en pro de la mitigación de la deserción escolar.

PALABRAS CLAVE: Análisis, factores, deserción escolar, nivel superior.

\section{ABSTRACT}

This article presents a documentary analysis on desertion, as well as the causes that generate it in higherlevel institutions. The databases used were Science Direct, Scielo, Redalyc, Google Academic, Latindex, obtaining a sample of 53 scientific documents identified with the keywords "school dropout". Among other factors, four key variables were considered within the analysis, such as: conceptualization of school dropout, description of possible factors of school dropout, characterization of the factors with the greatest impact on school dropout, programs and methodologies to address and reduce school dropout; Through the document review process, the information was delimited to extract only the most prominent one contained in different sources. The results allowed to determine the factors that are immersed in school dropout and how these factors are part of a materialized risk at the moment that a student decides to truncate their education, that is why as a conclusion of this analysis it is proposed that the institutions create detection mechanisms and implement modifications to their study programs, including teaching strategies and tutorial follow-ups in order to mitigate school dropouts.

KEY WORDS: Analysis, factors, school dropout, higher level.

\section{INTRODUCCIÓN}

De acuerdo con Diaz-Camargo (2020) "la educación es uno de los pilares fundamentales que garantizan el desarrollo de un país” (p. 2). Sin embargo, una condición que impacta en la calidad de la formación de ciudadanos es la deserción escolar; la cual, es una problemática que afecta a estudiantes de todo el mundo, de ahí que sus consecuencias impacten a la sociedad en su conjunto (Rochin, 2021).

\footnotetext{
${ }^{1}$ Profesora del Tecnológico Nacional de México, Campus La Chontalpa, rocio.gr@chontalpa.tecnm.mx

${ }^{2}$ Profesor del Tecnológico Nacional de México, Campus La Chontalpa, stalin.gm@chontalpa.tecnm.mx 
Por lo anterior, resulta necesario definir qué se entiende por deserción escolar. La palabra deserción significa desertar o abandonar. En el ámbito educativo se le conoce comúnmente como el abandono escolar, que corresponde a una decisión personal o por causas de diversos factores, que imposibilita al educando a continuar con la formación, lo que ocasiona poco interés o desmotivación en continuar en el proceso de aprendizaje (Hernández, Álvarez \& Aranda, 2017). Estos eventos generan preocupación, debido a que imposibilitan una formación académica exitosa. En el mismo contexto, Barradas-Arenas (2020) afirma que "el abandono escolar, en México, es un problema que el sistema educativo ha sido incapaz de resolver" (p. 36).

En este orden de ideas, la deserción, definida desde un punto de vista individual, hace referencia a todas aquellas metas y propósitos provenientes de las personas que se incorporan al sistema de educación, debido a que una gran variedad de objetivos y proyectos caracterizan las intenciones de los alumnos y las alumnas que ingresan a una institución educativa. En donde, algunos no se identifican con la graduación o no son precisamente compatibles con los objetivos y proyectos de la institución elegida. Más todavía, existe la posibilidad de que las metas no sean perfectamente claras para el individuo que se inscribe en la universidad y modifica su postura o decisión a lo largo de su trayectoria académica como estudiante.

Por lo tanto, conceptualizar la deserción a partir de un punto de vista personal, es más difícil que un fácil registro de las metas o de los fines con que cada individuo ingresa al sistema de enseñanza preeminente. Esto, debido a que en muchas ocasiones la deserción se vincula con el hecho de que las experiencias de una persona, en una determinada institución, son percibidas internamente como un fracaso o como lo que se proponía concretar en ella (Tinto, 1982).

Es en este mismo sentido que, el concepto "deserción" está mejor aplicado, puesto que comprende a una sociedad de intereses entre el individuo, que ingresa en una institución de educación superior, y los observadores externos que se proponen incrementar la retención de estudiantes. Por lo que, para quienes dirigen la institución en especial, esta situación representa un fracaso de la propia organización; la cual, no ayudó al alumno a conseguir lo que originalmente buscaba al ingresar en la universidad. Para Marchesi Ullastres (2003) el término fracaso escolar "transmite la idea de que el alumno "fracasado" no ha progresado prácticamente nada durante sus años escolares en el ámbito de sus conocimientos ni en su desarrollo personal y social" (p. 7).

Es entonces que, el problema de la deserción cambia cuando nos trasladamos de los aspectos institucionales hacia los más altos organismos educativos que los sustentan. A partir del punto de vista estatal, ejemplificando, los abandonos que involucran transferencias entre instituciones del estado tienen la posibilidad de no significar deserciones, en el sentido preciso del término, a diferencia de los traslados hacia las instituciones privadas o ubicadas fuera del estado, donde es posible que dichos abandonos sean considerados como deserciones.

En consecuencia de lo descrito y en consideración de que el análisis documental "consiste en el análisis de diversas teorías, a través de un conjunto de operaciones de orden intelectual y mecánico" (Salazar-Gómez \& Tobón, 2018, p. 3), este estudio centra su atención en la producción documental y al conocer su existencia, se posesiona de ella, la asimila mediante 
idiomas documentales construidos por medio de claves y normas, útiles para ordenar las fuentes de forma que facilite su implementación.

Por lo anterior, es que en el presente trabajo se reportan los resultados de un análisis documental; para el cual, se determinaron las siguientes 4 metas o categorías de análisis: 1. Conceptualización de la deserción escolar, 2. Descripción de posibles factores de deserción escolar, 3. Caracterización de los factores de mayor impacto en la deserción escolar y 4. Programas y metodologías para abordar y disminuir la deserción escolar. Por lo tanto, se busca determinar las características de los factores de mayor impacto en la deserción escolar, plasmando alternativas, posibles programas y metodologías para abordar y disminuir la deserción escolar.

\section{METODOLOGÍA}

La metodología que se utilizó en el presente análisis corresponde a una investigación con enfoque cualitativo-descriptivo, donde se realiza una revisión literaria. El procedimiento que se utilizó fue contrastar y sintetizar la información que se organizó en diversas categorías que abordan desde el marco conceptual hasta el contexto de la deserción escolar, con el objetivo de "buscar, seleccionar, organizar y analizar un conjunto de materiales escritos para responder una o varias preguntas sobre un tema" (Tobón, et al., 2015, como se citó en Hernández- Ayala et al., 2016, p. 401); el cual, se realiza mediante un registro documental, "este último consiste en buscar citas textuales y no textuales que se toman de diversas fuentes para hacer análisis, críticas, comparaciones, contrastes en ideas, conceptos y metodologías" (Martínez-Corona y Palacios-Almón, 2019, p. 183).

Para el análisis documental se determinaron cuatro categorías, las cuales se pueden apreciar en la Tabla 1.

Tabla 1. Análisis de categorías empleadas en el estudio. Fuente: Elaboración propia.

\begin{tabular}{|c|c|}
\hline Categorías & Pregunta Central \\
\hline $\begin{array}{l}\text { 1. Conceptualización de la deserción } \\
\text { escolar. } \\
\text { 2. Descripción de posibles factores de } \\
\text { deserción escolar. } \\
\text { 3. Caracterización de los factores de } \\
\text { mayor impacto en la deserción } \\
\text { escolar. } \\
\text { 4. Programas y metodologías para } \\
\text { abordar y disminuir la deserción } \\
\text { escolar. }\end{array}$ & $\begin{array}{l}\text { 1. ¿Qué es la deserción escolar? } \\
\text { 2. ¿Cuáles son los posibles factores } \\
\text { de deserción escolar? } \\
\text { 3. ¿Qué características tienen los } \\
\text { factores de mayor impacto en la } \\
\text { deserción escolar? } \\
\text { 4. ¿Qué programas y metodologías } \\
\text { se pueden abordar para disminuir } \\
\text { la deserción escolar? }\end{array}$ \\
\hline
\end{tabular}

Para el desarrollo del estudio se realizaron búsquedas en bases de datos científicas bajo la guía de las siguientes palabras esenciales: "deserción escolar", "educación superior" "deserción en México" y "scholar dropout". Para la selección de documentos se siguieron los siguientes criterios: 1) Que fueran artículos, libros y capítulos de libros de las siguientes bases de datos: Science Direct, Scielo, Redalyc, Google Académico, Latindex; 2) Que se publicaran entre 2016 y 2021; 3) Que aborden uno o varios elementos de las categorías establecidas, y 4) Que su enfoque integrara características tanto de investigación cualitativa 
como cuantitativa (Posada González, 2017, p. 239). En la Tabla 2, se muestra el concentrado de los documentos consultados en el presente análisis, mediante una síntesis cuantitativa de los seleccionados.

Tabla 2. Documentos analizados. Fuente: Elaboración propia (datos del análisis).

\begin{tabular}{|l|c|l|c|c|}
\hline Documento & $\begin{array}{l}\text { Acorde al } \\
\text { tema }\end{array}$ & $\begin{array}{l}\text { De } \\
\text { conceptualización }\end{array}$ & $\begin{array}{l}\text { Latinoamerican } \\
\text { os }\end{array}$ & $\begin{array}{l}\text { De otras } \\
\text { regiones }\end{array}$ \\
\hline Teóricos & 28 & 10 & 34 & 4 \\
\hline Empíricos & 11 & & 7 & 1 \\
\hline Libros & 3 & & 2 & \\
\hline Manuales & 1 & & 1 & \\
\hline
\end{tabular}

\section{RESULTADOS}

Antes de iniciar la descripción de los resultados a nivel categoría, se resalta lo expresado por Romo (2014):

"En la actualidad, la tutoría se reconoce como la mejor estrategia para apoyar al alumno en el desarrollo de una metodología de estudio y de trabajo apropiado a su nivel educativo. Se ofrece apoyo y supervisión en temas complejos de las diversas asignaturas, se procura crear un clima de confianza que permita conocer aspectos de la vida personal del alumno, que influyen directa o indirectamente en su desempeño y se le señalan y sugieren actividades extracurriculares que favorezcan su desarrollo integral" (p. 2).

\section{Categoría 1. Conceptualización de la deserción escolar.}

"El problema de la deserción escolar en México a nivel superior es bastante preocupante" (Mireles Campuzano et al., 2019, p. 4), ya que, según Valenzuela (2018), es un factor que incide en los índices de eficiencia terminal en todos los niveles educativos. Por lo tanto, son términos directamente relacionados y es una situación que afecta a estudiantes en todos los contextos de la enseñanza superior.

Para Huerta et al., (2016), "el abandono y suspensión de los estudios escolares posteriores se deben a causas familiares, económicas, desmotivación y falta de credibilidad en la escuela; así como, falta de centros de estudios superiores en la misma zona" (p. 34). En concordancia con esta idea, el Departamento Nacional de Estadística (DANE) (2016) señala que la deserción se produce cuando el estudiante se desvincula definitiva o temporalmente del sistema educativo formal.

En congruencia con lo anterior, Huerta, Velasco y Jiménez (2016) expresan que para el estudio de la deserción escolar se deben considerar diversos factores como la escolaridad de los padres y la condición de convivencia con sus hijos; así como ciertos problemas relacionados con la desmotivación, el desinterés por la escuela y las adicciones o la violencia. Por tanto, si bien es cierto que, la deserción corresponde a una decisión personal, también forma parte de un conjunto de factores que influyen directamente en la alteración de los 
estudios, ya que puede ser forzada o influenciada de forma positiva o negativa (Garzón \& Gil, 2016).

Ahora, al definir el término deserción, se observa en diferentes investigaciones que su concepción ha ido cambiando según avanza el tiempo, asociándose a diferentes enfoques e investigadores. Así, se conoce a la deserción no solo como la situación en la que los estudiantes abandonaban sus estudios en cualquier momento de su trayectoria académica; sino que, esta decisión, que altera la continuidad de los estudios, puede darse de manera parcial o permanente por una renuncia motivada, ya sea voluntaria o forzosa, abordada como una decisión de índole personal voluntaria, vinculada o influenciada por intereses personales o ajenos del propio estudiante (Chong González, 2017).

Abello et al., (2016) definen la deserción como un acto voluntario de renuncia originado por intereses personales pero que también puede estar asociado a características propias de la institución. Por su parte, Garzón \& Gil (2016) entienden a la deserción como "la disminución en el número de estudiantes desde el inicio hasta el final del curso, programa, institución o sistema bajo revisión" (p. 308), siendo esta última, la definición más completa en torno al concepto de la deserción.

\section{Categoría 2. Descripción de posibles factores de deserción escolar}

Para caracterizar los posibles factores que inciden en la deserción escolar, se identifica como relevante la afirmación de que "es un hecho que la deserción universitaria es un fenómeno urgente por atender, debido al alarmante porcentaje de desertores, el cual cambia según la universidad ya sea pública o privada, el programa de estudio, el entorno socioeconómico, el ambiente educativo, el ambiente familiar, entre otros muchos factores" (Mireles Campuzano et al, 2019, p. 5).

Adicionalmente a las condiciones de cambio, se reconoce que también influyen factores sociales y culturales de la familia, las relaciones entre iguales y aspectos de tipo económico. En forma particular, "algunos de los factores familiares, como las expectativas de logro académico de los padres, el apoyo emocional y económico", han sido algunos de los factores señalados por Huerta et al., (2016, p. 37) como los relevantes, donde destaca que el $23 \%$ de ellos no cuenta con posibilidades económicas.

Con respecto a los estudios sobre deserción en la educación universitaria, Cabanzo (2017, como se citó en Castillo-Sánchez et al., 2020), afirma que estos se han centrado en los problemas de los estudiantes. En esta condición, desde un punto de vista social, económico y cultural, se brindan características de estos grupos y de su comportamiento; el cual, varía de acuerdo con la carrera, la complejidad de los cursos matriculados, el entorno de aprendizaje propio e institucional y la convivencia, entre otros aspectos. El autor señala que entre los factores asociados a esta problemática destacan las siguientes:

- Diferencias entre los enfoques curriculares, las condiciones de las instituciones de la educación media y la formación recibida por estudiantes en este nivel educativo, que provoca desigualdades en las condiciones al ingresar a la educación en una institución de educación superior y su capacidad para afrontar los retos de este nivel educativo.

- Deficiencias en la formación matemática previa del estudiantado. 
- Débil vinculación de la matemática del currículo universitario del estudiante con otras disciplinas o carreras.

- Clases universitarias desarrolladas con base en un libro de texto que limita la investigación, creatividad de estudiantes y docentes, así como su actualización e interés por la materia.

- Una mala percepción estudiantil con respecto a la matemática, lo que provoca rechazo hacia ella y, por ende, hacia las carreras y cursos que incluyen este componente en su currículo.

Visto desde otra perspectiva, si bien es posible que un determinado grupo de estudiantes cuente con las mismas condiciones, si la carrera y la complejidad de los cursos matriculados varía, puede existir un grado mayor de deserción; esto no significa que dichos factores son necesariamente codependientes. En concreto, la deserción puede ser vista como el resultado la toma de decisiones derivada de un conjunto de diferentes factores que influyen en el estudiante.

Categoría 3. Caracterización de los factores de mayor impacto en la deserción escolar. Respecto a este tema, se pueden encontrar diversos estudios bibliográficos vinculados con el fracaso escolar, que se centran en las dificultades de aprendizaje o en el tratamiento del rechazo escolar y los factores de riesgo asociados al ausentismo (García-Fernández et al., 2016). En los estudios se logran identificar las causas de deserción de estudiantes universitarios más significativas, donde las variables personales de análisis que se presentan con mayor frecuencia son: la edad, el género y el estado civil; en la variable socio familiar, se han realizado estudios para determinar la deserción por el lugar de procedencia, composición familiar, nivel de educación que alcanzaron los padres y su nivel socio cultural; en lo que respecta al factor académico, los estudios se enfocan al nivel de rendimiento académico y número de materias tomadas por ciclo o periodo. Sin embargo, se aprecia que en estos estudios no existe un criterio que englobe todas las posibles causas de deserción estudiantil (Bazantes, Ruiz y Álvarez, 2016; Álvarez y López, 2017; ChongGonzález, 2017; Castillo-Sánchez, 2020).

Es importante mencionar que, la pandemia originada por el coronavirus causante de la enfermedad COVID-19 ha provocado cambios sin precedentes en el mundo. El año 2020 provocó una conmoción a escala planetaria; por lo que, es fácil de comprender que los sistemas educativos no estuviesen preparados para afrontar la situación (Cáceres-Correa, 2021). De manera inmediata, fue necesario cambiar a un modelo de educación virtual o a distancia, para lo cual el sistema educativo no estaba adaptado.

La Encuesta para la Medición del Impacto COVID-19 en la Educación en México (ECOVID-ED) (INEGI, 2020), revela que las clases a distancia generaron gastos emergentes. Un poco más del $28 \%$ de las viviendas con población de tres a 29 años, hizo un gasto adicional para adquirir teléfonos móviles inteligentes; el $26.4 \%$ contrató el servicio de Internet fijo y el $20.9 \%$ para adquirir mobiliario como sillas, mesas, escritorios o adecuar espacios para el estudio.

Esta situación es de alta relevancia para la deserción; ya que, el porcentaje de la población que no concluyó el ciclo escolar debido a la pandemia por COVID-19 en educación superior fue de $44.6 \%$, el $17.1 \%$ porque tenía que trabajar, $8 \%$ por falta de dinero, mientras que $30.3 \%$ 
mencionó otras razones. Dentro de los motivos asociados a este fenómeno, por los cuales los alumnos concluyeron los estudios se destacan los siguientes: se "perdió el contacto con maestras/maestros o no pudo hacer tareas $(28.8 \%)$, alguien de la vivienda se quedó sin trabajo o redujeron sus ingresos $(22.4 \%)$, la escuela cerró definitivamente $(20.2 \%)$ y carecía de computadora, otros dispositivo o conexión a internet (17.7\%)" (INEGI, 2020, p. 12). En este mismo sentido, según los resultados que presenta el INEGI, se puede afirmar, que la presencia del COVID-19 potenció el número de casos de deserción escolar.

Categoría 4. Programas y metodologías para abordar y disminuir la deserción escolar. En México, "reducir los altos niveles de abandono y aumentar los índices de eficiencia terminal, en todos los subsistemas de la educación media superior y superior, continúa siendo un reto para las autoridades educativas" (SEP, 2020, p. 199); por lo que, es un desafío definir las estrategias que permitan a las instituciones de educación superior superar los altos índices de deserción, reprobación y rezago, al igual que los bajos índices de eficiencia terminal (Sánchez et al., 2020). Es por lo que, el programa institucional de tutorías (PIT) tiene gran importancia en la búsqueda de la reducción de la deserción escolar.

La tutoría académica es el camino para detectar de forma apropiada los inconvenientes académicos que muestran los alumnos y que están afectando de manera directa a la eficiencia terminal de los programas educativos; por consiguiente, la reestructuración del seguimiento de las trayectorias estudiantiles se inicia con la evaluación a partir del punto de vista del tutor, con la identificación y asentimiento de los indicadores de trayectoria académica personal.

De acuerdo con Dávila Martínez \& López Pérez (2019) “el objetivo principal de un sistema de tutorías deben ser los estudiantes, se deben cuidar algunos aspectos principalmente: su entorno social, familiar, económico, de salud, las referidas a la preparación previa y las que tienen que ver con la adquisición de las capacidades necesarias para avanzar en el conocimiento" (p. 3). Lo que implica que este programa es de alta relevancia institucional y su presencia representa una estrategia para mitigar efectos asociados a los puntos de atención.

En lo que respecta a la educación superior, de acuerdo con Ramos-Betancourt et al. (2019), la tutoría "atiende la necesidad de potenciar la formación integral de los estudiantes y uno de sus principales retos, consiste en lograr que una proporción elevada de alumnos alcance niveles de desempeño académico favorables para culminar satisfactoriamente sus estudios en los plazos previstos" (p. 81). Esta situación implica una serie de retos a las IES.

En este sentido, el trabajo tutorial implica un acompañamiento al alumno desde que ingresa a la institución hasta su egreso, incluyendo la titulación; esto, al considerar que la calidad de los programas educativos está directamente relacionada con el desempeño escolar de cada uno de los estudiantes y el tiempo en que concluyen su preparación profesional. Sin embargo, al dar un servicio individual y personalizado, es importante considerar las características, condiciones y expectativas que tienen los estudiantes al realizar su trayectoria académica (UNACAR, 2018).

Por lo anterior, todas las personas que conforman la institución educativa deben interesarse por los problemas que enfrentan los estudiantes y atenderlos mediante todo tipo de actividades que permitan crear un ambiente efectivo de integración (Donoso, Donoso y Arias, 2018). En este sentido, Cruz Nieto \& Perusquía de Carlos (2016), señalan que son diversas las estrategias y modalidades que llevan a cabo las instituciones para dar cumplimiento al 
programa de tutorías en favor de los alumnos, entre las que se pueden mencionar la tutoría grupal, individual o entre pares.

Es por lo que, se enfatiza sobre el proceso de tutorías, ya que puede utilizarse como una herramienta viable, que permita no solo disminuir la deserción, sino también mejorar el aprovechamiento escolar, estimular las destrezas de los estudiantes y apoyar su formación integral.

\section{CONCLUSIONES}

La información analizada permitió caracterizar los factores mencionados; sin embargo, es necesario ahondar más en los siguientes estudios, con el propósito de obtener un análisis más robusto que integre un mayor número de variables. Cabe resaltar que, con base en los resultados del registro documental se propone que las instituciones con índices de deserción elevados implementen acciones que les permitan mitigar referido indicador; así como, dar seguimiento a los programas que brindan acompañamiento y/o apoyo a los estudiantes en distintas áreas (tutorías).

En conclusión, es de vital importancia nutrir el presente estudio integrando especificaciones en torno a la decisión de desertar, a fin de clasificarla, por ejemplo, de acuerdo con la tipología propuesta por Páramo y Correa (1999) e identificar cuál es el tipo de deserción que es más relevante en la institución educativa y, a su vez, cuáles son las variables que explicarían el fenómeno (González \& Arizmendi, 2018).

\section{BIBLIOGRAFÍA}

Abello, R., Vila, I., Lagos, I., Espinoza, C., \& Diaz, A. (2016). Identidad de aprendiz como herramienta analítica de experiencias universitarias en el contexto de la permanencia y el abandono: propuesta teórica. PAIDEIA (58), 11-34. ISSN 07164815

Álvarez, P., \& López, D. (2017). Estudios sobre deserción académica y medidas orientadoras de prevención en la Universidad de la Laguna (España). Revista Paradigma, 38(1), 48-71.

Amaya-Amaya, A., Huerta-Castro, F., \& Flores-Rodríguez, C. O. (2020). Big Data, una estrategia para evitar la deserción escolar en las IES. Revista iberoamericana de educación $\quad$ superior, 11(31), 166-178, DOI: https://doi.org/10.22201/iisue.20072872e.2020.31.712

Barradas-Arenas, U., Hernández-Chan, G., Soto-Valenzuela, A., \& Canto-Rodríguez, J. (2020). Aplicación móvil de apoyo para la disminución del ausentismo escolar en la educación media superior. Revista Internacional de Investigación e Innovación Tecnológica, 7(42). 35-52, ISSN 2007-9753

Bazantes, Z., Ruiz, M., \& Álvarez, M. (2016). Deserción estudiantil universitaria en ecuador y su fluencia en la calidad del egresado. (U. T. Ecuador., Ed.) Revista Magazine de las Ciencias. ISSN 2528-8091.

Cabanzo, E. (2017). Las matemáticas y su influencia en la deserción universitaria. Universidad Militar Nueva Granada, Colombia.

Cáceres-Correa, I. (2021). Acerca de la escolaridad a distancia y la deserción en Pandemia. 
Utopía y Praxis Latinoamericana, 26(2), 11-12. ISSN: 1315-5216

Castillo-Sánchez, M., Gamboa-Araya, R., \& Hidalgo-Mora, R. (2020). Factores que influyen en la deserción y reprobación de estudiantes de un curso universitario de matemáticas. Uniciencia, 34(1), 219-245. DOI: https://doi.org/10.15359/ru.34-1.13

Chong González, E. G. (2017). Factores que inciden en el rendimiento académico de los estudiantes de la Universidad Politécnica del Valle de Toluca. Revista latinoamericana de estudios educativos, 47(1), 91- 108. ISSN: 0185-1284

Cruz Nieto, H. G., \& Perusquía de Carlos, I. (2016). Tutorías entre pares como estrategia de apoyo en la evaluación sumativa.

Dávila Martínez, J. M., \& López Pérez, Z. M. (2019). Impacto del Programa Institucional de Tutorías en la deserción del Programa de Ingeniería Civil de la Universidad Autónoma de Zacatecas. Investigación Científica, 13(2). 7-7

Departamento Nacional de Estadística (DANE) (2016). Departamento Administrativo Nacional de Estadísticas. Disponible en http://www.dane.gov.co/index.php/estadisticas-por-tema/educacion

DGEST. (2012). Manual del Tutor del SNIT. México. Obtenido de http://www.dgest.gob.mx/images/areas/docencia/2012-

1/tutor/MANUAL_DEL_TUTOR.DD.pdf

Díaz-Camargo, E., Riaño-Garzón, M., Ballesteros García, S. A., Mora Vallejo, D. Y., Soto Méndez, L. I., Yolima Valero, I., \& Bautista-Sandoval, M. J. (2020). Acompañamiento psicológico y permanencia en estudiantes universitarios. Psicología Escolar e Educacional, 24. Disponible en https://www.scielo.br/j/pee/a/5kMt6CrcVRdffFKNhHPpK9v/?format=html

Donoso, S., Donoso, G. y Arias, Ó. (2018). Iniciativas de retención de estudiantes de educación superior. Calidad en la Educación, (33), 15-61. DOI: http://dx.doi.org/10.31619/caledu.n33.138

García-Fernández, J.M.; Inglés, C.J.; Gonzálvez, C.; Vicent, M.; Delgado, B. Y GómezNúñez, M.I. (2016). Revisión bibliométrica del rechazo escolar: perspectivas de investigación y su análisis. Educativo Siglo XXI, 34 (1), 71-92. DOI: https://doi.org/10.6018/j/252541

Garzón, A., \& Gil, J. (2016). El papel de la procrastinación académica como factor de la deserción universitaria. Revista Complutense de Educación. 28(1). 307-324. ISSN: 1130-2496

González Catalán, Felipe Ignacio, \& Arismendi Vera, Karin Jeanette. (2018). Deserción Estudiantil en la Educación Superior Técnico-Profesional: Explorando los factores que inciden en alumnos de primer año. Revista de la educación superior, 47(188), 109-137. ISSN 0185-2760

Hernández - Ayala, Hernán y Tobón-Tobón, Sergio y (2016), Análisis documental del proceso de inclusión en la educación. Ra Ximhai, 12(6), 399-420. ISSN: 16650441.

Hernández, M., Álvarez, J., \& Aranda, A. (2017). El problema de la deserción escolar. 
Revista Internacional de Ciencias Sociales y Humanidades, SOCIOTAM, 27(1), 89-112. Disponible en https://www.redalyc.org/pdf/654/65456040007.pdf

Huerta, P., Velasco, M. y Jiménez, M. (2016). Causas de la deserción escolar en las telesecundarias de la zona 55. Revista Huella de la Palabra, (8). DOI: https://doi.org/10.37646/huella.vi8.491

INEGI. (2020). Encuesta para la Medición del Impacto COVID-19 en la Educación (ECOVID-ED) 2020. México. Disponible en https://www.inegi.org.mx/contenidos/investigacion/ecovided/2020/doc/ecovid_e d_2020_nota_tecnica.pdf

Marchesi Ullastres, A. (2003). El fracaso escolar en España. Madrid, España: Fundación Alternativas.

Martínez-Corona, J. I., \& Palacios-Almón, G. E. (2019). Análisis de la Gestión para Resultados en el Marco de la Sociedad del Conocimiento. Atenas. Revista Científica Pedagógica, 3(47), 180-197.

Mireles Campuzano, L. E., Alarcón Romano, M., Campos Rivera, J. C., Maldonado Aguilar, J. M., \& Pérez Gerónimo, F. d. (2019). Modelo de gestión académica para disminuir la deserción de los estudiantes de nuevo ingreso del Tecnológico Nacional de México en Celaya. Pistas Educativas 40(132).

Posada González, N. L. (2017). Algunas nociones y aplicaciones de la investigación documental denominada estado del arte. Investigación Bibliotecológica, 31(73), 237-263. ISSN 2448-8321

Ramos-Betancourt, I., Amaro-Martinez, J., \& Rodarte-Borrego, B. E. (2019). La función de la tutoría en la universidad. Ciencias de la conducta y humanidades - Proceedings T-I, 80-102.

Rochin Berumen, F. L. (2021). Deserción escolar en la educación superior en México: revisión de literatura. Revista Iberoamericana para la Investigación y el Desarrollo Educativo, 12(22). DOI: https://doi.org/10.23913/ride.v11i22.821

Romo A (2014). Los programas institucionales de Tutoría: actores, procesos y contextos. ANUIES: México.

Salazar-Gómez, E., \& Tobón, S. (2018). Análisis documental del proceso de formación docente acorde con la sociedad del conocimiento. Revista Espacios, 39(53), 17.

Sánchez Martínez, F. E., Barrera Cámara, R. A., \& Canepa Sáenz, A. (2020). Estudio evaluativo del Sistema Institucional de Tutorías para el seguimiento de trayectorias escolares. RIDE. Revista Iberoamericana para la Investigación y el Desarrollo Educativo, 10(20). ISSN 2007-7467

Secretaría de Educación Pública. (2020). Programa Sectorial de Educación 2020-2024. México. Disponible en: https://www.gob.mx/cms/uploads/attachment/file/562380/Programa_Sectorial_de _Educaci_n_2020-2024.pdf

Tinto, V. (1982). Defining dropout: A matter of perspective. New Directions for Institutional Research, 1982(36), 3-15. 
Tobón, S., Gonzalez, L., Salvador Nambo, J., \& Vazquez Antonio, J. M. (2015). La socioformación: un estudio conceptual. Paradigma, 36(1), 7-29.

Universidad Autónoma del Carmen (UNACAR). (2018). Modelo Educativo Acalán. Campeche, México: http://www.unacar.mx/Dir_General_Academica/Documentos/Modelo_Educativo_A calan/Modelo_Ed_Acalan2017.pdf.

Valenzuela López, P. (2018). Deserción académica en la Universidad Tecnológica de Hermosillo y su incidencia en la eficiencia terminal. REAXION, 6(1). Disponible en:http://reaxion.utleon.edu.mx/Art_Desercion_academica_en_la_Universidad_T ecnol\%C3\%B3gica_de_Hermosillo_y_su_incidencia_en_la_eficiencia_terminal. html 